\title{
EL AMBIENTE EN PRISIÓN: \\ LA ATENCIÓN RECIBIDA POR LAS RECLUSAS \\ Y LAS RELACIONES INTRAMUROS ${ }^{1,} 2$
}

\section{THE ENVIRONMENT IN PRISON: \\ THE CARE PROVIDED TO THE WOMEN PRISIONERS \\ AND INTRAMURAL RELATIONS}

\section{O AMBIENTEA PRISÃO: \\ O ATENDIMENTO PRESTADO ÀS MULHERES PRESAS \\ E AS RELAÇÕES INTRAMUROS}

\author{
María del Mar García-Vita
}

UnIVERSIDAD DE GRANADA, EsPAÑA

Miguel Melendro Estefanía

Universidad Nacional de Educación a Distancia, España

RESUMEN: La vida cotidiana de los centros penitenciarios es merecedora de estudios que indaguen sobre la diversidad de interacciones que se producen dentro de los muros de prisión. Esas interacciones determinarán en gran medida cuál es el clima que rige los centros y las posibilidades que nos ofrece el medio penitenciario en cuanto al tratamiento de los reclusos y reclusas.

En nuestra investigación sobre mujeres reclusas y los procesos de reinserción, hemos advertido algunas particularidades que pueden ser de utilidad en su tratamiento. Se trata de una investigación de ámbito nacional, con una muestra estratificada de 599 reclusas en segundo y tercer grado de cumplimiento (se han obtenido 538 cuestionarios válidos y 61 entrevistas) y de 36 profesionales. La información fue recogida durante el año 2011 mediante un cuestionario y entrevistas semiestructuradas a reclusas, junto a un cuestionario a profesionales del medio penitenciario.
El análisis de la información recogida nos ha hecho constatar cómo incide la organización del personal penitenciario, su relación con las reclusas, junto a las relaciones creadas dentro de prisión, en el ambiente existente en los centros penitenciarios y en el proceso reeducador propio de instituciones penitenciarias. Se muestra así mismo cómo y en qué aspectos la estancia en prisión puede ser un factor positivo, potenciador de las condiciones sociales, personales y emocionales que facilitarán la reinserción de las reclusas y su retorno a la vida en libertad.

PALABRAS CLAVE: Prisión; sexo femenino; relaciones humanas; educación social; profesionales del medio penitenciario; asistencia al detenido.

ABSTRACT: The daily life of prison deserves studies that investigate the diversity of interactions that take place within its walls. These interactions largely 
determine the climate that governs the centers and the possibilities offered by the prison of the treatment of inmates of both sexes.

In our research on women prisoners and reintegration processes, we have noticed some peculiarities that may be useful in treatment. This is a national research, with a stratified sample of 599 inmates in second and third degree of compliance (we have obtained 538 valid questionnaires and 61 interviews) and 36 professionals. The information was collected in 2011 using a questionnaire and semi-structured interviews of inmates, along with a questionnaire for professionals of prisons.

The analysis of the information gathered has caused us to observe the influence of the organization of prison personnel, their relationship with women prisoners, along with the relationships formed in prison, in the existing environment in prisons and the re-education process itself of prisons. It likewise shows how and in what ways the stay in prison can be a positive factor, enhancing the social, personal and emotional conditions that will facilitate the reintegration of prisoners and their return to life in freedom.

KEY WORDS: Prison; female; human relations; community education; professionals from prison; prison welfare.

RESUMO: O cotidiano da prisão merece estudos que investigam a diversidade de interações que ocor- rem dentro dos muros da prisão. Estas interações determinam em grande parte o que os centros climáticos que regem e as possibilidades oferecidas pela prisão sobre o tratamento de prisioneiros de ambos os sexos.

Em nossa pesquisa sobre mulheres presas e processos de reintegração, temos notado algumas peculiaridades que podem ser úteis no tratamento. Esta é uma pesquisa nacional, com uma amostra estratificada de 599 detentos em segundo e terceiro grau de cumprimento (obtivemos 538 questionários válidos e 61 entrevistas) e 36 profissionais. As informações foram coletadas em 2011 através de um questionário e os presos entrevistas semi-estruturadas, juntamente com um questionário para prisões profissionais.

A análise da informação recolhida nos fez observar como isso afeta a organização de prisão, a sua relação com mulheres presas, juntamente com as relações formadas na prisão, no ambiente existente em prisões e reeducação próprio processo de prisões. Do mesmo modo, mostra como e de que maneira a estadia na prisão pode ser um fator positivo, aumentando a. Social, pessoal e emocional que irá facilitar a reintegração de prisioneiros e seu retorno à vida em liberdade.

PALAVRAS CHAVE: Prisão; sexo feminino; relações humanas; educação comunitária; profissionais deprisão; protecção a reclusos.

\section{Introducción}

La prisión como institución -y su concepción como espacio para la retención de personas- ha ido evolucionando hasta convertir en su objetivo central la rehabilitación y reeducación de los individuos que allí residen. Pero, a pesar de lo positivo de este planteamiento, hay quienes consideran que la modalidad de organización en las cárceles responde aún a lo que Goffman (1972) denominó "instituciones totales o cerradas", marcadas por el control de los sujetos y la deconstrucción de su yo y de los roles y capacidades personales que les eran útiles para la vida diaria en el exterior.La particularidad del contexto al que nos referimos supone en sí una referencia importante para posibilitar la transformación de los procesos que se producen en prisión, ya que como señala Clemente (1997a, p. 324), “la prisión es más que la suma de actividades ocupacionales que dentro de ella se realizan [...] la prisión misma moldea e integra las actividades que se producen dentro de ella". Así, el conocimiento del ambiente en prisión y las relaciones que se establecen en su interior tendrán un papel clave a la hora de valorar hasta qué punto se ha producido esa transformación de la cárcel, de su conceptualización como institución total a su rol rehabilitador y reeducador.

[ 44 ] Ma DEL MAR GARCÍA-VITA, MIGUEL MELENDRO ESTEFANÍA

SIPS - PEDAGOGIA SOCIAL. REVISTA INTERUNIVERSITARIA [1139-1723 (2013) 22, 43-56] TERCERA ÉPOCA 
A las particularidades estructurales del propio sistema, habrá que añadir las disfunciones presentes en la realidad penitenciaria y otros factores condicionantes como la sobrepoblación reclusa, la escasez de personal y las consecuencias que derivan de ellos (Aranda y Rivera, 2012). Esto añade dificultades a los objetivos y propuestas rehabilitadoras, que deberían suponer un principio inspirador y transversal a la vida en la cárcel y que habrían de priorizarse para evitar una mayor alienación de los presos y presas, organizando la vida en prisión en similitud a la vida exterior, mediante la promoción de los derechos de los presos y de sus relaciones exteriores (De la Cuesta y Blanco, 2001).

En este sentido, Del Rey (2004) valora que la labor de tratamiento realizada intramuros no aprovecha adecuadamente dos estrategias básicas a tener en cuenta en un modelo de intervención en prisión: construir un ambiente idóneo que facilite el tratamiento e implicar a todo el personal que de una forma u otra trabaja con los internos. En la construcción de ese ambiente idóneo en el centro, las relaciones interpersonales suponen un elemento central. Hemos de tener en cuenta, al analizarlas, que se trata de relaciones marcadas por el contexto y por el particular microclima social de una institución cerrada y, por tanto, caracterizada por condiciones y factores diferentes a los que determinan las interacciones en la vida exterior (Clemente, 1997b). Las relaciones que se producen a diario dentro de los centros penitenciarios se configuran a partir del estatus social creado en ese contexto y de los roles que se ejercen en él. Así, se producen relaciones claramente diferenciadas entre funcionarios, de éstos con los internos/as, entre las personas recluidas y entre todos ellos dependiendo de su sexo.

La investigación realizada, base de este artículo, aborda de forma específica estas cuestiones, en el marco de los procesos de reinserción de las mujeres reclusas y de la elaboración de unas pautas de actuación socioeducativas específicas acordes a las características del colectivo. Para ello resulta crucial identificar los problemas y necesidades de las mujeres en prisión y, entre ellos, los que vienen determinados por el ambiente que reina en los centros penitenciaros, la estructura organizativa y de control que marca la vida cotidiana y las rutinas penitenciarias, como elementos relevantes de nuestro trabajo de investigación.

\section{Cotidianeidad y vida penitenciaria}

Al observar la realidad de las cárceles españolas comprendemos que allí se siguen procesos de socialización diferentes a los comunes; internos e internas se adaptan a la vida bajo normas y preceptos que marcan una gran diferencia con la sociabilidad extramuros. Una sociabilidad, la de prisión, que configura relaciones sociales únicas, donde la "promoción social" se basa en aspectos que son insignificantes en la vida exterior (Viegas, 2009).

Para su comprensión son necesarios algunos detalles de la rutina penitenciaria. Así, el horario y calendario de actividades para el régimen ordinario 3 es aprobado por el Consejo de Dirección del centro, aunque se garantizan ocho horas de descanso nocturno, un mínimo de dos horas para asuntos propios y tiempo suficiente para atender a las actividades culturales y terapéuticas y a los contactos con el mundo exterior (Reglamento Penitenciario, 1996; art. 77). Se determina que en la organización de esos horarios participen los reclusos y reclusas (art. 55). En régimen abierto 4 (art. 85) cada interno/a sigue distintas pautas relativas a las salidas al exterior y diferentes modalidades de vida según las regulaciones de la Junta de Tratamiento para cada caso. No obstante, se dispone de un horario y unas reglas a cumplir durante la estancia en el centro. Especial consideración se tiene en este aspecto con las internas en Unidades Dependientes de Madres, en función de sus necesidades familiares (art. 179).

Las características de la vida cotidiana de las personas privadas de libertad coinciden, según Goffman (1972), con el principio institucional de la confinación de personas para su vigilancia. De acuerdo con este principio, la intimidad y la oportunidad de cambio de escenario para las distintas tareas diarias -dormir, divertirse y trabajar- se limitan de forma importante, al igual que resulta obligatoria la participación en la programación establecida en el centro. También es albergada esta idea, en alusión específica a la situación de las mujeres recluidas, en el Informe Nacional de la Fundación SURT (Asociación de Mujeres para la Inserción 
Laboral) sobre Mujeres, integración y prisión (Cruells e lgareda, 2005) destacando que esta situación desprovee a las reclusas de capacidades vitales, provoca falta de autonomía, iniciativa, responsabilidad y capacidad de decisión, haciendo que su progreso en prisión dependa de su capacidad de adaptación a las normas de la institución penitenciaria -obediencia, buena conducta y participación en las actividades- siendo más beneficioso para ellas y su situación penitenciaria una mayor sumisión y acatamiento de las normas.

En relación a ese proceso de adaptación hay que hacer alusión al fenómeno de la "prisionización", término acuñado por Clemmer (1958), con el que pretende describir la repercusión de la subcultura carcelaria en todas las personas que están en prisión (personas encarceladas y trabajadores/as). Las consecuencias de este proceso implican a toda persona que haga vida dentro de prisión y esto pudiera explicar los comportamientos tan similares entre vigilantes y reclusos/as, extendiéndose al funcionariado los efectos del proceso de internalización bajo un reglamento y unos preceptos para la convivencia que le son impuestos en su rol laboral (González Salas, 2001). Entre las consecuencias que acontecen sobre la identidad social de los internos en centros penitenciarios, Trillo (2008) menciona la pérdida de vinculaciones con el exterior, la adaptación al entorno y la creación de nuevos vínculos interpersonales.

Además de los rasgos generales que rodean la reclusión de las personas, tenemos que mencionar una serie de aspectos específicos y casuísticas diversas que convergen en prisión.

El género, como elemento clave de nuestro estudio, requiere de referencias específicas. El ser mujer en un lugar creado para varones y en inferioridad numérica a ellos, repercute de diversas maneras y, en lo respectivo al tratamiento, se traduce en que no siempre existan módulos destinados a albergarlas y, por lo tanto, con frecuencia no sea posible clasificarlas -por edades o situación penal- para ofrecerles recursos diferenciados. Así, son las madres reclusas con sus hijos menores de tres años en la cárcel, las únicas que siempre disponen de un espacio propio para su internamiento (Igareda, 2007). Del mismo modo, los recursos que se destinan a las mujeres son menores que los destinados a los hombres, ya que disponer de recursos formativos, laborales y de tratamiento específicos para mujeres resulta más costoso. Es destacable además el hecho de que esos programas socioeducativos, formativos o de tratamiento específicos de mujeres sean limitados en cuanto a temática, en su mayoría vinculados al mundo doméstico y la ética del espacio privado, y que incluyan muy escasamente la perspectiva de género (Aguilera, 2011; Cruells e lgareda, 2005; Pérez Rodríguez, 2010), siendo habitual que sean los peor remunerados y previamente rechazados por las cárceles masculinas (Almeda, 2002). Aguilera (2011) por su parte sustenta la idea de que a las presas se les aplican de modo indiscriminado medidas de control y vigilancia existentes en las prisiones de hombres, sin que estas medidas se ajusten al peligro real que representa la población femenina. Por añadidura, la separación de hombre y mujeres en los centros provoca que el disfrute de los espacios comunes no siempre sea equitativo entre ambos colectivos, teniendo las mujeres el acceso más restringido a espacios como polideportivos, bibliotecas, enfermerías, etc.

Otro de esos aspectos que no podemos obviar es el de las drogodependencias y los consumos por parte de reclusos y reclusas, que también están fuertemente condicionados por el contexto penitenciario. Moreno (1999) ha estudiado la variable motivación para consumir drogas y ha concluido que los motivos para consumir drogas son diferentes en prisión que en situación de libertad. Los motivos por los que se consume en prisión se basan en la rutina y monotonía que existe en ella, así como en la misma condición de reclusión. Se plantea, por lo tanto, que una modificación de las condiciones de esta cotidianeidad en determinado sentido, provocaría la disminución de los motivos para consumir en prisión.

Finalmente, entre los estándares que definen el ambiente predominante en prisión, según Ruíz Pérez (2006), el número de personas reclusas es determinante, estando una mayor densidad asociada a niveles más negativos en el clima de las relaciones, ya que ese hecho frecuentemente limita el acceso a aquellos recursos más escasos en prisión, como el acceso a algunos talleres, trabajos, programas... Según datos de García-Guerrero y Marco (2012), en el año 2011 la ocupación penitenciaria total en nuestro país se situaba en torno al 135\%. El hacinamiento -albergar un mayor número de reclusos para los que está preparado el centro- de acuerdo con el Centro Internacional para Estudios Penitenciarios (2005), produce consecuencias como,

[ 46 ] Ma DEL MAR GARCÍA-VITA, MIGUEL MELENDRO ESTEFANÍA

SIPS - PEDAGOGIA SOCIAL. REVISTA INTERUNIVERSITARIA [1139-1723 (2013) 22, 43-56] TERCERA ÉPOCA 
entre otras, el deterioro de la salud mental de las personas recluidas y la creación de un ambiente más proclive a la peligrosidad, tanto para el personal penitenciario como para los internos e internas.

\section{Personal penitenciario y organigrama de funcionamiento}

Diversos autores (Ayuso, 2001; Redondo, 2004; Redondo, Pozuelo y Ruíz, 2007) han reparado en que los Centros Penitenciario se enfrentan al reto de conciliar objetivos contradictorios: el mantenimiento del orden y la seguridad, y el fin del tratamiento. En ocasiones, ambos entran en conflicto debido a que la consecución de los objetivos del tratamiento colisiona con un medio en el que las actividades humanas, hasta las más básicas, están reglamentadas y controladas (Ayuso, 2001). Debido a esta dualidad existen figuras profesionales propias del régimen penitenciario y aquellas propias del tratamiento penitenciario, siendo necesaria una coordinación entre ambas (Redondo et al., 2007).

Cuando nos referimos a la atención que reciben los/as reclusos/as nos referiremos a la totalidad de la acción ejercida sobre ellos por el medio penitenciario, pero con especial consideración a la acción directa que se implementa en los internos a través de la vertiente del tratamiento. Por ello, pensamos que es importante clarificar los órganos internos que convergen en un Centro Penitenciario. Según el Reglamento Penitenciario vigente de 1996, Título XI, Capítulo 2, los órganos colegiados que organizan el funcionamiento de los centros son la Junta de Dirección, la Junta de Tratamiento -que tiene a su disposición el Equipo o Equipos Técnicos necesarios-, la Comisión Disciplinaria y la Junta Económica-Administrativa.

Entre estos espacios profesionales, uno de los que más nos interesa en relación a la temática de este artículo es el del Equipo Técnico, que vendría a componerse y organizarse en función de las especificidades y necesidades del centro, pudiendo estar formado hasta por once figuras: un Jurista, un Psicólogo, un Pedagogo, un Sociólogo, un Médico, un Ayudante Técnico Sanitario/Diplomado universitario en Enfermería, un Maestro o Encargado de Taller, un Educador, un Trabajador Social, un Monitor Sociocultural o Deportivo, un Encargado de Departamento. Entre sus cometidos está el trabajo directo con los internos/as. Son el colectivo dedicado a la acción, mientras que la Junta de Tratamiento se ocupa principalmente de la toma de decisiones y de establecer las pautas de acción.

El trabajo interprofesional e interdisciplinar, con un grupo tan amplio y tan diverso de profesionales interviniendo en prisión es ciertamente complejo. Como afirma Domínguez (1997), el carácter multiprofesional, sobre todo de los Equipos de Tratamiento, puede conllevar conflictos -prejuicios grupales, estereotipos, modelos teóricos distintos, formación diferente, distintas categorías profesionales y posibilidades de promocionar, dificultades de planificación y relación, etc.- entre grupos corporativos, junto al temor de todos a perder autonomía, recursos y autoridad. A ello hay que añadir las diferencias y el posible alejamiento del colectivo de vigilancia y el de tratamiento. Todo esto hace que el personal del medio penitenciario tenga que afrontar un difícil reto en la coordinación efectiva de sus actuaciones y, si ésta no se realiza adecuadamente, sufra el riesgo del desencanto o la fatiga en la tarea a desarrollar. En este sentido plantea Ayuso (2001) la importancia del estudio de los empleados públicos del medio penitenciario, ya que son los profesionales que tratan directamente con los reclusos, con la característica añadida de que son los empleados públicos con mayor porcentaje de bajas laborales de tipo psiquiátrico en toda la Administración Pública española.

En relación a ello debemos volver a mencionar el fenómeno de la "prisionización" y su efecto sobre el funcionariado. González Salas (2001) señala cómo el funcionario de vigilancia sufre las consecuencias del proceso de internalización, de un reglamento y una manera de convivir impuestas, muy distantes de las que le ofrece su entorno social en libertad. A esto se añade que los funcionarios de prisiones desempeñan una tarea de notable dificultad, que puede exigir una implicación emocional continuada y no exenta de conflictividad. En este contexto tan peculiar, el estudio de fenómenos como el burnout o "queme" en el trabajo y el incumplimiento de las expectativas que ambas partes (empleado y empleador) depositan en la relación laboral, es ineludible (Topa y Morales, 2005). El síndrome burnout está muy presente en el terreno de los profesionales del medio penitenciario, siendo objeto de diversos estudios en los últimos años que han con-

EL AMBIENTE EN PRISIÓN: LA ATENCIÓN RECIBIDA POR LAS RECLUSAS Y LAS RELACIONES INTRAMUROS [ 47 ] 
templado principalmente tres dimensiones: el cansancio emocional, la realización personal y la despersonalización. El elevado cansancio emocional de los funcionarios de vigilancia descrito en el estudio realizado por Hernández, Fernández, Ramos y Contador (2006) se relaciona con su labor de confrontación con los internos y los altos niveles de tensión emocional que produce. Por otro lado, se ve reforzado por el carácter de sus tareas, sencillas, monótonas y poco motivantes y por un elevado índice de despersonalización.

Todo ello se ve reflejado, como se indica en un reciente estudio llevado a cabo por Valderrama Bares (2012) en las percepciones de reclusos acerca del tratamiento. En ese estudio se visibiliza la necesidad de que los miembros de la junta de tratamiento mantengan más contactos con los presos, siendo ésta una premisa importante en la labor educativa durante el proceso de internamiento. Como veremos más adelante, una necesidad que se extiende a los componentes de los Equipos Técnicos de los centros penitenciarios.

\section{Las relaciones de los internos/as en prisión}

Como sostiene Cubilla (2011), lo cotidiano se reformula en base a las relaciones que en cada espacio social se dan, por lo que para acercarnos a la realidad diaria que viven los presos y presas, será necesario profundizar en el conocimiento de estas relaciones.

“En cada espacio social, en cada escenario, el hombre se reconoce en su carácter de ser social como co-constructor de competencias axiológicas, es decir, en su capacidad de cooperación en la construcción y reconstrucción de valores sociales en los diversos escenarios donde éste desarrolla, en el curso de su vida, infinitas relaciones con los demás individuos." (p. 1).

Las relaciones de las personas encarceladas deben ser abordadas distinguiendo dos escenarios: el de las relaciones externas (con familiares, con el sistema judicial...) y el de las relaciones internas (con otros presos/as, con familiares dentro de prisión, con los profesionales de la institución penitenciaria...). Si bien el desarrollo de unas relaciones equilibradas en ambos escenarios por parte de los/las reclusos/as son indispensables para el bienestar y condiciones de vida en prisión (García de Cortázar y Gutiérrez Brito, 2012), hasta ahora se han potenciado más los vínculos con el exterior, con notoria presencia de los estudios sobre la maternidad vivida en prisión en el caso de las mujeres presas, dejando como aspecto secundario y de menor interés la exploración de las interacciones que se producen dentro de prisión, que constituyen la parte central del trabajo que aquí aportamos.

Cuando hablamos de relaciones entre presos/as estamos partiendo de relaciones mayoritariamente pautadas por el sistema (García de Cortázar y Gutiérrez Brito, 2012) y marcadas por criterios espacio/temporales -funcionamiento del centro y pautas de vida- y de género -división por sexos en los espacios del centro. Clemente (1997a) apunta que la adaptación a un entorno tan característico y alejado de la vida en el exterior como el de prisión, provoca actitudes de dominio y/o sumisión en las relaciones interpersonales, ya sea por la necesidad de autoafirmarse agresivamente frente a la institución o para defenderse de ella. Los reclusos/as tienden a agruparse para tener una mayor fuerza, ya que sienten que la institución no tiene capacidad para proporcionarles seguridad, y así se generan relaciones de poder intrapenitenciarias (Trillo, 2008). Estas relaciones de poder entre los reclusos vienen marcadas por tres criterios: el tipo de delito cometido -especial valor otorgado a los delitos como atracos, estafas o robos, que requieren cierta sofisticación-, la experiencia delincuencial y carcelaria -mayor prestigio cuanto más tiempo de condena cumplida- y la conducta en prisión, de acuerdo al sistema de valores imperante en la cárcel (Clemente, 1997a).

En cuanto a la cultura carcelaria y el código de los internos, Ruíz Pérez (2009) menciona la necesidad de diferenciar entre sus características si hablamos de hombres o de mujeres. En el caso de las reclusas, se establece una tendencia a constituir el grupo de acuerdo a la estructura familiar tradicional que prevalece en la sociedad libre, con una figura central que adquiere el rol materno -a modo de "madre" o "abuela"- y que es reconocida como líder. Ruíz Pérez y algunos de sus estudiantes han observado así mismo, en sus estancias en prisión, que la cohesión entre las internas tiene un importante componente socioafectivo y de apoyo.

[ 48 ] Ma DEL MAR GARCÍA-VITA, MIGUEL MELENDRO ESTEFANÍA

SIPS - PEDAGOGIA SOCIAL. REVISTA INTERUNIVERSITARIA [1139-1723 (2013) 22, 43-56] TERCERA ÉPOCA 
Por otra parte, un fenómeno habitual en el perfil prototípico de las internas, derivado de su necesidad de afecto tras el frecuente abandono de la familia de la que provienen, es la búsqueda de pareja dentro de prisión, lo que reduce su sentimiento de soledad emocional (Carcedo, López y Orgaz, 2006).

\section{Metodología}

La investigación de la que procede la información que reflejamos en este trabajo tiene como tema central el estudio de la situación de las mujeres en prisión, y sus procesos de reinserción. En concreto, se plantea como objetivo central el estudio de la situación de las mujeres del medio penitenciario español y su relación con los procesos socioeducativos de reincorporación a la vida social.

Se trata de una investigación de ámbito nacional, con una muestra estratificada de 599 reclusas en segundo y tercer grado de cumplimiento, internas o en medio abierto, (un 15\% de la población reclusa femenina en el momento del trabajo de campo), y de 36 profesionales. La media de edad de las participantes es de 36 años, estando la mayoría de ellas entre los 25 y los 49 años. La nacionalidad de las reclusas entrevistas es para un $69 \%$ la española, siendo el $31 \%$ restante extranjeras, entre las que cabe distinguir el colectivo de mujeres de América Latina 21,6\%.

La información fue recogida en el año 2011 mediante 3 instrumentos: un cuestionario con ítems abiertos y cerrados, un total de 538 cuestionarios válidos a mujeres (para un nivel de confianza del 95,5\% y un error muestral del $\pm 3,9$ puntos); una entrevista semiestructurada con un total de 61 testimonios recogidos, y un cuestionario sobre 43 programas socioeducativos en prisión, para cuya cumplimentación se ha contado con la participación de 36 profesionales del medio penitenciario. Se visitaron un total de 42 centros de toda la geografía española. La participación era voluntaria, tras haberles explicado el objeto del estudio que se estaba llevando a cabo y establecer un consentimiento informado para el correcto tratamiento de los datos y su confidencialidad más allá de la investigación. La información ha sido tratada con el programa estadístico IBM SPSS (versión 15 y 20) y los datos de tipo cualitativo se han analizado a partir de la emergencia de categorías significativas y su triangulación.

A continuación se aportan los resultados de aquellos elementos de la investigación relacionados con el tema central de este trabajo: el ambiente en prisión, la atención recibida por las reclusas y las relaciones intramuros. En concreto se aportan resultados destacados de nueve ítems del cuestionario a mujeres reclusas, cinco ítems de la entrevista a mujeres reclusas y tres ítems del cuestionario de profesionales 5 .

\section{Análisis e interpretación de resultados}

Cómo ya hemos referido anteriormente, para poder interpretar adecuadamente la dinámica de prisión es necesario profundizar en el conocimiento de las relaciones interpersonales relativas a los miembros de la comunidad penitenciaria, y más concretamente de las relaciones intramuros, tema que ha sido muy escasamente abordado en investigación. Nos hemos centrado, por tanto, en la indagación acerca de cómo se configuran las relaciones diarias de las personas que habitan dentro de prisión, pero siendo conscientes de la importancia que tienen los recuerdos, sensaciones y características de su pertenencia a estructuras familiares y sociales externas.

Entre los factores a analizar para tal fin se encuentran las valoraciones que las reclusas otorgan a la función realizada por los distintos profesionales, sus relaciones con ellos, la naturaleza de los contactos con los compañeros/as de internamiento y con las demás figuras institucionales y el voluntariado que forman parte de la vida en prisión. Del mismo modo, se han recogido datos sobre la percepción que los profesionales tienen de cómo es acogido su trabajo por parte de las internas.

Un primer resultado de la investigación nos muestra cómo la segmentación del trabajo del personal penitenciario hace que éste sea valorado por las internas de muy distintas formas. La casuística es diversa, ya que hay internas que requieren de la atención de ciertos profesionales y otras no, o que han tenido 
mayor contacto, o un contacto más directo o íntimo con unos que con otros en función de sus circunstancias.

La mayor valoración de unas figuras profesionales que otras se ha asociado en nuestro estudio tanto a la frecuencia con que se encuentran con estos profesionales como a la calidad de la relación mantenida. Muy clarificadores resultan testimonios del tipo:

"Bien, es que tampoco hablo mucho... a la directora no la conozco y con los funcionarios y eso no hablo mucho, vamos lo necesario." (EX E312)

"Psicólogos vienen pocos por aquí. Educadores los que más vienen. Nunca tuve ningún problema con ellos." (NA E2O3)

De esta forma, los funcionarios/as de vigilancia, maestros/as, educadores/as y monitores/as poseen mejores valoraciones -ya que no solo han sido valorados por más mujeres, sino también de forma más positivaque los psicólogos/as, juristas, trabajadores/as sociales, o cargos ejecutivos de la prisión, de acuerdo a los datos mostrados en la Tabla 1. Por otra parte, el escaso contacto que las reclusas tienen con algún grupo concreto de profesionales, ha incidido en su decisión de no valorar esas figuras.

Tabla 1. Valoración de las relaciones con profesionales (en \% de respuestas sobre total de respuestas válidas en cada caso)

\begin{tabular}{|c|c|c|c|c|c|c|c|}
\hline & $\begin{array}{c}\mathrm{N} \\
\text { (respuestas } \\
\text { válidas)* la }\end{array}$ & $\begin{array}{l}\% \text { que } \\
\text { representa en } \\
\text { la muestra total }\end{array}$ & $\begin{array}{l}\text { Relación } \\
\text { no } \\
\text { valorada*** }\end{array}$ & Mala & Regular & Buena & $\begin{array}{l}\text { Muy } \\
\text { buena }\end{array}$ \\
\hline Con funcionarios (hombres) & 514 & $95,5 \%$ & $12,7 \%$ & $1,9 \%$ & $15,4 \%$ & $56,4 \%$ & $13,6 \%$ \\
\hline Con funcionarias (mujeres) & 520 & $96,7 \%$ & $6,5 \%$ & $3,3 \%$ & 18,1 & $54,2 \%$ & $17,9 \%$ \\
\hline Trabajadores sociales (hombres) & 479 & $89 \%$ & $35,5 \%$ & $1,7 \%$ & $9,6 \%$ & $40,3 \%$ & $12,9 \%$ \\
\hline Trabajadoras sociales (mujeres) & 508 & $94,4 \%$ & $12 \%$ & $2,8 \%$ & $15,7 \%$ & $51,8 \%$ & $17,7 \%$ \\
\hline Educadores (hombres) & 501 & $93,1 \%$ & $76,4 \%$ & $1,4 \%$ & $10,4 \%$ & $52,3 \%$ & $79,5 \%$ \\
\hline Educadoras (mujeres) & 477 & $88,7 \%$ & $27, \%$ & $2,9 \%$ & $10,5 \%$ & $43,6 \%$ & $15,9 \%$ \\
\hline Psicólogos (hombres) & 472 & $87,7 \%$ & $43,2 \%$ & $1,7 \%$ & $8,9 \%$ & $34,1 \%$ & $12,1 \%$ \\
\hline Psicólogas (mujeres) & 477 & $88,7 \%$ & $26,4 \%$ & $1,9 \%$ & $11,1 \%$ & $43,4 \%$ & $17,2 \%$ \\
\hline Juristas (hombres) & 466 & $86,6 \%$ & $47,6 \%$ & $3,2 \%$ & $12,7 \%$ & $29 \%$ & $7,5 \%$ \\
\hline Juristas (mujeres) & 465 & $86,4 \%$ & $48,6 \%$ & $3 \%$ & $10,1 \%$ & $30,1 \%$ & $8,2 \%$ \\
\hline Monitores (hombres) & 457 & $84,9 \%$ & $42,6 \%$ & $0,4 \%$ & $5,5 \%$ & $36,5 \%$ & $14,9 \%$ \\
\hline Monitoras (mujeres) & 460 & $85,5 \%$ & $35,7 \%$ & $0,4 \%$ & $6,5 \%$ & $38,9 \%$ & $18,5 \%$ \\
\hline Educadores infantiles (hombres) & 125 & $23,2 \%$ & $83,3 \%$ & - & $1,2 \%$ & $11,4 \%$ & $4,1 \%$ \\
\hline Educadoras infantiles (mujeres) & 423 & $78,6 \%$ & $79,9 \%$ & - & $2,6 \%$ & $12,1 \%$ & $5,4 \%$ \\
\hline Subdirectores (hombres) & 456 & $84,8 \%$ & $58,3 \%$ & $1,1 \%$ & $6,1 \%$ & $27,4 \%$ & $7 \%$ \\
\hline Subdirectoras (mujeres) & 464 & $86,2 \%$ & $56,5 \%$ & $2,2 \%$ & $7,1 \%$ & $26,7 \%$ & $8,2 \%$ \\
\hline Directores (hombres) & 459 & $85,3 \%$ & $60,8 \%$ & $1,7 \%$ & $5,9 \%$ & $24,8 \%$ & $6,8 \%$ \\
\hline Directoras (mujeres) & 458 & $85,1 \%$ & $62,9 \%$ & $1,5 \%$ & $4,8 \%$ & $22,1 \%$ & $8,7 \%$ \\
\hline Maestros (hombres) & 469 & $87,2 \%$ & $42,4 \%$ & $0,2 \%$ & $3,8 \%$ & $32,4 \%$ & $21,2 \%$ \\
\hline Maestras (mujeres) & 473 & $87,9 \%$ & $34,9 \%$ & $0,6 \%$ & $4,7 \%$ & $34,7 \%$ & $25,2 \%$ \\
\hline Voluntarios (hombres) & 457 & $84,9 \%$ & $36,3 \%$ & - & $3,1 \%$ & $32,8 \%$ & $27,8 \%$ \\
\hline Voluntarias (mujeres) & 464 & $86,2 \%$ & $28,9 \%$ & $0,2 \%$ & $3 \%$ & $35,3 \%$ & $32,5 \%$ \\
\hline
\end{tabular}

* Número de respuestas válidas en cada ítem

** Muestra total $=538$ 
Otro dato relevante obtenido es el que se refiere a las relaciones que las reclusas mantienen de forma diferenciada con los funcionarios y con las funcionarias. En un $70 \%$ de los casos estas relaciones son valoradas como buenas o muy buenas, sin que exista una diferencia significativa en función del sexo. Debemos matizar que, cuando se les ha preguntado por los motivos que impulsan esas relaciones positivas con el personal penitenciario, la ambigüedad está presente, y en un porcentaje importante de casos la buena actitud hacia ese personal busca la consecución de los intereses propios ( $45 \%$ de las reclusas encuestadas), mientras que el rechazo de esta actitud es menos habitual (29\%).

Por otra parte, declaran las mujeres de nuestro estudio no haber tenido problemas con el personal penitenciario en un $80 \%$ de los casos, y solo un $17 \%$ manifiesta haberlos tenido en alguna ocasión. Cerca de un $10 \%$ de reclusas no manifiestan buenas relaciones con los/as funcionarios/as pero tampoco admiten haber tenido ningún problema concreto con ellos/as. Las entrevistas, por otra parte, dan información más específica, ya que se les interroga sobre la tipología de esos problemas; entre ellos destacan las agresiones físicas o verbales, el incumplimiento de compromisos y atenciones que han sido solicitadas y no cubiertas.

En general, las reclusas describen relaciones positivas con las personas con las que tratan y conviven en prisión. Especialmente son muy buenas las referidas a los compañeros de internamiento varones con un $53,1 \%$ de respuestas en este sentido. Con ellos mantienen una relación en muchas ocasiones distante, a través de cartas y con escaso contacto físico, y no exenta de cierta idealización. Una posible explicación a este dato, procedente de las entrevistas a reclusas, tiene que ver con esa idealización de la figura masculina a la vista de sus necesidades afectivas, dentro del contexto de encierro y soledad al que están sometidas y a la particular situación, generalizada en su colectivo, de abandono por parte de las figuras más representativas, sobre todo de sus parejas en el exterior de la prisión. Aunque debemos mencionar la existencia de una alta cifra de mujeres que dicen no poder valorar esas relaciones con sus compañeros varones en prisión, bien porque no existen $(20,1 \%)$ o porque no quieren entran a valorarlas $(18,3 \%)$ debido a la segregación y falta de interacción que existe en la cotidianeidad entre los dos sexos.

Las relaciones entre compañeras también son valoradas muy positivamente (80,5\%). En el cuestionario a reclusas se aborda la percepción que las mujeres tienen respecto cuestiones relativas a esas relaciones. La proporción de reclusas que refieren sentimientos de rivalidad, envidia o competitividad entre compañeras es de un 85,3\%; quienes reconocen la existencia de discusiones acaloradas alcanza el 43,9\%, con el matiz de que esas discusiones por problemas o conflictos les hacen "llegar a las manos" en un 22,7\% de los casos. Pero, al mismo tiempo, un 93\% de estas mismas mujeres interpreta que en el módulo es colaboradora con sus compañeras $y$, cuando lo necesita, les ayuda, bien sea siempre o en ocasiones más o menos frecuentes.

Gráfico 1. Comparativa de respuestas sobre la relación con las compañeras de prisión (\% sobre respuestas válidas)

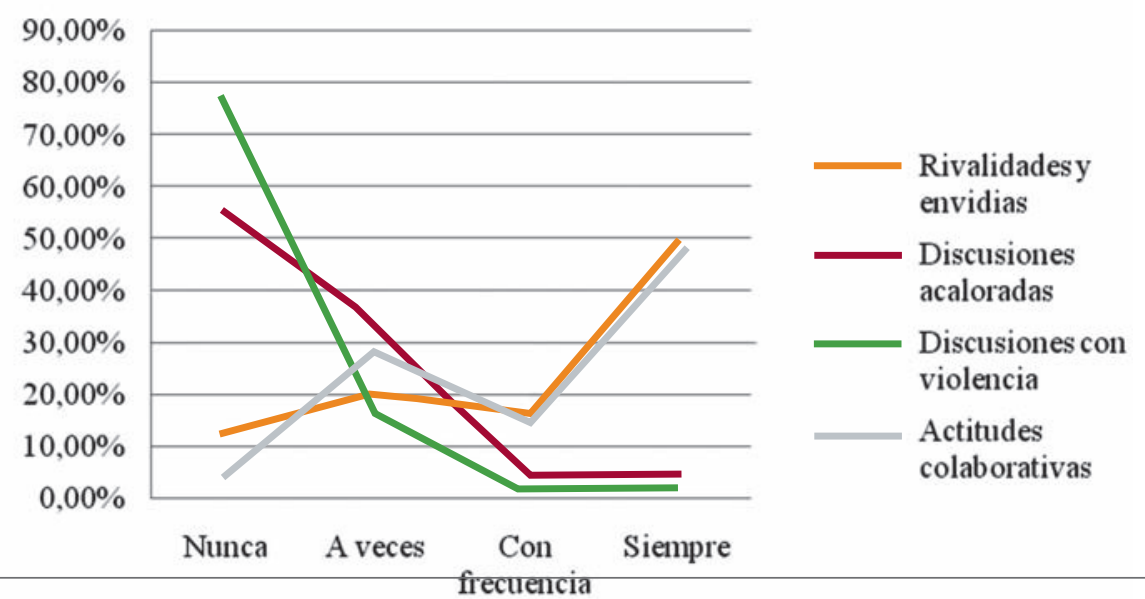

EL AMBIENTE EN PRISIÓN: LA ATENCIÓN RECIBIDA POR LAS RECLUSAS Y LAS RELACIONES INTRAMUROS [ 51 ] SIPS - PEDAGOGIA SOCIAL. REVISTA INTERUNIVERSITARIA [1139-1723 (2013) 22, 43-56] TERCERA ÉPOCA 
Con ello se desmitifica la creencia generalizada de que las rivalidades entre mujeres prevalecen frente a las relaciones de tipo solidario ya que las internas valoran, en términos generales, tener buenas o muy buenas relaciones entre ellas, a pesar de las situaciones de conflicto de las que no está exenta la vida en prisión.

Por otra parte, se puede observar cómo las mujeres que sienten no haber tenido un apoyo fuerte o real antes de su entrada en prisión, manifiestan que sus relaciones interpersonales han aumentado y mejorado tras su ingreso en prisión. Así, antes de entrar en prisión un 6,7\% de las reclusas declaran no haber tenido apoyo de nadie, mientras que durante su estancia en prisión este porcentaje se reduce al 3,3\%. Es interesante también constatar cómo aunque el apoyo de la madre $(55,6 \%)$ sigue siendo el principal soporte de las mujeres presas, aparece la figura de las compañeras de internamiento ocupando un segundo lugar como soporte y apoyo ante dificultades, reconocido por el $54,1 \%$ de ellas.

Gráfico 2. Apoyo recibido durante el cumplimiento de la condena (\% en función del total de muestra)

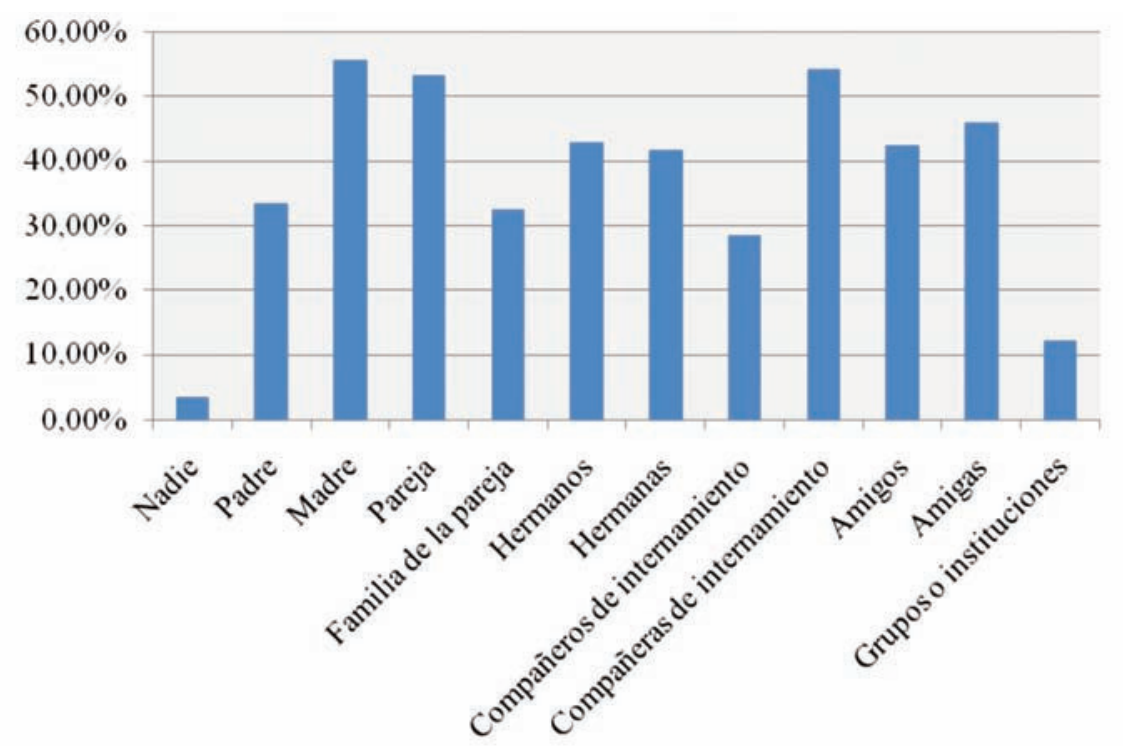

La prisión aparece pues como punto de inflexión en ese apoyo sociofamiliar. Son muy esclarecedores los testimonios recogidos en las entrevistas sobre, por ejemplo, la situación actual en que se encuentran sus relaciones familiares, con testimonios similares al de esta reclusa:

"Con mi madre las relaciones han mejorado. Le he contado todos mis problemas y me ha sabido entender. No sé. Siento que tengo un apoyo en ella. La verdad es que sí. He recuperado bastante mi relación con ella." (EX E409)

Por último, también se ha sondeado a los profesionales de tratamiento que coordinan programas sobre las características y cuestiones de funcionamiento de éstos. Las respuestas a una de las preguntas, sobre la implicación de las mujeres en los programas de tratamiento, respaldan la idea de que existe un nivel alto de participación ( $90,7 \%$ de los profesionales dicen que son muy o bastante participativas) y que asisten con regularidades hasta finalizar las actividades ( $88,3 \%$ ), siendo un $37,2 \%$ de estos profesionales proclives a pensar que hay escasez de plazas en sus actividades.

\section{Discusión y conclusiones: la realidad intramuros y la posibilidad de aprovechar las relacio- nes que allí se producen}

El panorama descrito arroja luz sobre una parte de la realidad penitenciaria muy poco estudiada hasta el momento, aunque si mencionada parcialmente en algunos estudios (Ayuso, 2001; Clemente, 1997a, 1997b; Cruells

[ 52 ] Ma DEL MAR GARCíA-VITA, MIGUEL MELENDRO ESTEFANÍA

SIPS - PEDAGOGIA SOCIAL. REVISTA INTERUNIVERSITARIA [1139-1723 (2013) 22, 43-56] TERCERA ÉPOCA 
Gráfico 3. Estrategias básicas en la intervención intramuros (adaptado a Del Rey 2004)
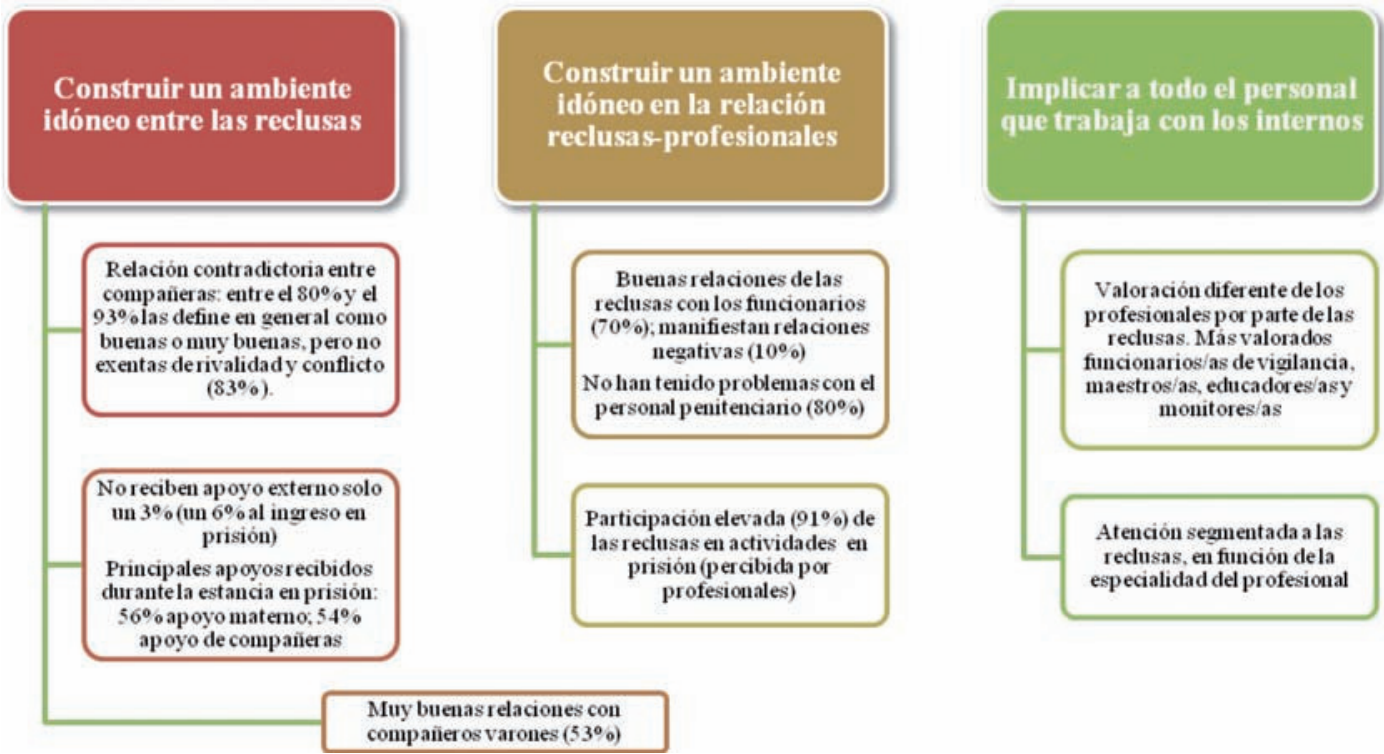

e Igareda, 2005; Del Rey, 2004; García de Cortázar y Gutiérrez Brito, 2012; Moreno, 1999; Ruíz Pérez, 2006, 2009; Trillo, 2008; Viegas, 2009; entre otros) y nos permite avanzar en el esclarecimiento de la dinámica y el cariz que adquiere la vida dentro de los centros de penitenciarios.

El carácter multiprofesional presente en el mundo penitenciario y los posibles problemas derivados de ello -dificultades en el uso coordinado de recursos escasos, posturas distintas según disciplina y formación de cada profesional o dificultades de organización-, a los que hace referencia Domínguez (1997) y tal y como se refleja en nuestra investigación, provoca que con más frecuencia de la deseada las reclusas reciban una atención parcelada, que atiende a sus necesidades vitales de forma fragmentada y no global, obedeciendo más a los estándares de lo que el mundo académico y laboral ha creído conveniente: separar la atención a las reclusas en función de las distintas disciplinas y funciones laborales. La intervención interdisciplinar y coordinada es, desde la percepción de las reclusas, minoritaria o, al menos, poco visible para ellas. Además, la propia organización del personal en distintos órganos de gobiernos y acción provocan que, como bien describe Valderrama Bares (2012), no siempre exista un trato directo de las reclusas con los directivos y Junta de Tratamiento, que son quienes tienen la función de tomar decisiones sobre su situación y condena siendo, en nuestra investigación, los profesionales que menos valoraciones reciben y en sentido más negativo. Estos factores repercuten de forma importante en el transcurso del proceso rehabilitador, pero no son los únicos. Hay otros elementos relevantes en la definición de la rutina penitenciaria.

En este sentido, de especial trascendencia es la socialización intramuros, que se perfila distinta a la que se produce en entornos en libertad, y se manifiesta en diversas cuestiones. Por un lado, se visibiliza en la actitud correcta de las presas y en el mantenimiento de unas buenas relaciones con el personal, en pro de conseguir beneficios penitenciarios o un mejor trato de éstos. Los intereses que les mueven a ello están relacionados con la cobertura de necesidades básicas como la alimentación, el descanso, el sueño, o la higiene personal, como ha definido Viegas (2009), algo que genera una importante dependencia del otro, y entre ellos ineludiblemente de los funcionarios/as. Si bien se concibe como una conducta inteligente la adaptación y el acatamiento de las normas establecidas para mejorar su situación en prisión, en paralelo se produce una situación de empobrecimiento personal de las reclusas, al dejar de ejercer capacidades vitales tales como la iniciativa, la responsabilidad y la autonomía (Cruells e Igareda, 2005).

A pesar de lo positivo del dato que obtenemos sobre las relaciones entre reclusas y el personal de prisión, no podemos obviar los episodios descritos por parte de ellas sobre agresiones verbales, algunas incluso 
físicas, y sobre todo su decepción y su queja al ver incumplidas las expectativas que tenían puestas en los funcionarios/as. Lo expuesto por multitud de autores acerca de la crudeza del trabajo en el medio penitenciario (Hernández et al., 2006; Topa y Morales, 2005) nos abre una puerta hacia posibles trabajos más concretos que relacionen el desgaste emocional y la falta de realización que soportan los trabajadores con estas percepciones de las reclusas.

Por otro lado, las relaciones que mantienen con los compañeros y compañeras de internamiento también permiten visibilizan el tipo de sociabilidad que se construye dentro de los muros de la prisión. Las reclusas, en su situación, no pueden establecer nuevos contactos íntimos a no ser con las personas con las que conviven dentro de prisión. Esta circunstancia tiene una especial relevancia, ya que viene unida a su pérdida de red social exterior y especialmente al distanciamiento de la pareja que nutre sus necesidades afectivas y con quien habrían de planificar en muchos casos su reinserción (Cruells e lgareda, 2005). Tanto cuando hablan de relaciones positivas con los reclusos varones, como cuando refieren no tener relación con ellos, se está poniendo de manifiesto la segregación espacial existente en prisión (García de Cortázar y Gutiérrez Brito, 2012) que marca las relaciones entre hombres y mujeres, y otorga connotaciones específicas a los vínculos que se crean entre ellos: frecuente creación de parejas (Carcedo et al., 2006) sin un conocimiento pleno del otro ni la posibilidad de contactos normalizados.

Un tercer tipo de relaciones a destacar son las originadas por la convivencia entre las mujeres. Esta convivencia es descrita por ellas como positiva, tendiendo a estructurarse de forma típica familiar (Ruíz Pérez, 2009) y ejerciendo un gran apoyo entre ellas. Tal y como exponen Clemente (1997a) y Trillo (2008), al afirmar que las relaciones entre compañeros de prisión están marcadas por el poder, por adquirir una situación de dominio o sumisión, encontramos su símil precisamente en la esa imitación de la estructura familiar, con una reproducción de la figura materna como cabeza del grupo de reclusas.

La realidad descrita nos muestra que, de forma generalizada, en la prisión existe un ambiente positivo, en base a las declaraciones de las mujeres con las que hemos trabajado. No obstante, no podemos centrarnos en el testimonio de una única parte de las implicadas. Las respuestas de las reclusas pueden estar desvirtuadas o darnos una información segmentada, sin duda una información subjetiva de su percepción de la realidad penitenciaria. Y, por otro lado, hay qué establecer que vínculo es realmente beneficioso para ellas y cuál no, con especial consideración a los contactos sentimentales o de pareja intrapenitenciarios, fomentados en gran medida, como hemos comentado anteriormente, por las carencias afectivas y de apoyo y la pérdida de red social.

Siendo cautos por tanto en la interpretación de la información obtenida, podemos avanzar la conclusión de que, en términos generales, las relaciones interpersonales, íntimas o de tipo profesional-usuario, son sanas, revestidas de un sentimiento de comprensión y apoyo. Algo especialmente destacado en la relación entre las compañeras de internamiento. Si este ambiente distendido es tal como lo han mostrado las mujeres que han participado en la investigación, nos ofrece un marco en el que esos vínculos positivos pueden ser aprovechados para su tratamiento y para mejorar las condiciones de su futura reinserción. Para ello creemos conveniente y muy indicado en la tarea de los equipos de tratamiento, profundizar en el análisis de las redes sociales que posee cada reclusa dentro de prisión, a fin de potenciar aquellas que tiene un efecto positivo sobre ellas y su adquisición de potencialidades de cara al regreso a la vida exterior.

\section{Referencias bibliográficas}

Aguilera, M. (2011). Mujeres en prisiones españolas. Crítica, 973, 44-48.

Almeda, E. (2002). Corregir y castigar. El ayer y hoy de las cárceles de mujeres. Barcelona: Bellaterra.

Aranda, M. y Rivera, I. (2012). Problemáticas detectadas en el sistema penitenciario español. Revista Crítica Penal y Poder, 3, 127-137. Recuperado de http://revistes.ub.edu/index.php/CriticaPenalPoder/article/view/3729

Ayuso, A. (2001). La intervención socioeducativa en el tratamiento penitenciario. Pedagogía Social. Revista Interuniversitaria, no6-7, 73-99.

[ 54 ] Ma del MAR GARCíA-VITA, MIGUEL MELENDRO ESTEFANÍA

SIPS - PEDAGOGIA SOCIAL. REVISTA INTERUNIVERSITARIA [1139-1723 (2013) 22, 43-56] TERCERA ÉPOCA 
Carcedo, R.; López, F. y Orgaz, M.B. (2006). Estudio de las necesidades socio-emocionales y sexuales de los presos. Boletín criminológico, 92, 1-4.

Centro Internacional para Estudios Penitenciarios (2005). Cómo resolver el hacinamiento en las prisiones (Nota Orientativa). Recuperado de http://www.prisonstudies.org/info/downloads.php?searchtitle=\&type=0\&month=O\&year=0\&lang=3\&author= \&search=Search

Clemente, M. (1997a). La organización social informal en la prisión. En M. Clemente y J. Núñez (coords.) Psicología Jurídica Penitenciaria II, (pp. 321-356). Madrid: Fundación Universidad Empresa.

Clemente, M. (1997b). Los efectos psicológicos y psicosociales del encarcelamiento. En M. Clemente y J. Núñez (coords.), Psicología Jurídica Penitenciaria II, (pp. 383-407). Madrid: Fundación Universidad Empresa.

Clemmer, D. (1958). The Prison Community. New York. Editorial: Rinehart \& Winston.

Cruells, M. e Igareda, N. (2005). MIP: Mujeres, integración y Prisión. Barcelona: Aurea Editories.

Cubilla, W. (2011). De las relaciones sociales intramuros. Márgenes. Espacio Pedagógico, 2. Recuperado de http://www.idaes.edu.ar/margenes/Ni/Cubilla__Articulo_VLNL_1.pdf

De la Cuesta, J. L. y Blanco, I. (2001). Nation Reports: Spain. En D. Zyl Smit y F. Dünkel (eds.), Imprisonment today and tomorrow: International Perspectives on Prisoners' Rights and Prison Conditions, (pp.609-634). La Haya: Kluwer Law International.

Del Rey, E. (2004). Intervención ambiental con drogodependientes encarcelados: principios, datos y líneas de actuación. Revista de estudios Penitenciarios, 250, 73-96.

Domínguez, F. (1997). La dirección y gestión de un equipo de observación y tratamiento. En M. Clemente y J. Núñez (coords.), Psicología Jurídica Penitenciaria II, (pp. 409-435). Madrid: Fundación Universidad-Empresa.

García de Cortázar, M. y Gutiérrez Brito, J. (2012). Relaciones en prisión. En C. del Val y A. Viedma (eds.), Condenadas a la desigualdad. Sistema de indicadores de discriminación penitenciaria, (pp. 143-172). Barcelona: Icaria.

García-Guerrero, J. y Marco, A. (2012). Sobreocupación en los centros penitenciarios y su impacto en la salud. Revista Española de Sanidad Penitenciaria, 14, 106-113.

Goffman, E. (2007). Internados. Ensayos sobre la situación social de los enfermos mentales. Buenos Aires: Amorrortu.

González Salas, A. E. (2001). Consecuencias de la prisionización. Revista Cenipec, 20, 9-22.

Hernández, L., Fernández, B., Ramos, F. y Contador, I. (2006). El síndrome de burnout en los funcionarios de Vigilancia Penitenciaria. International Journal of Clinical and Health Psychology, 6, 599-611.

Igareda, N. (2007). Mujeres en prisión. En A. I. Cerezo y E. García (coords.), La prisión en España: una perspectiva criminológica, (pp. 75-100).Granada: Comares.

Moreno, M. P. (1999). Situación de internamiento versus situación de libertad: diferencias en algunas variables en presos drogodependientes. Adicciones, 11 (1), 23-31.

Pérez Rodríguez, S. (2010). La igualdad y atención con las mujeres reclusas en Andalucía. En F. Del Pozo, F. Añaños, I. Mavrou y D. Sevilla (coords.) Educación, Salud y drogodependencias. Enfoques, programas y experiencias en ámbitos de exclusión (pp.12-29). Madrid: Produg Multimedia, S.L.

Redondo, S. (2004). Tratamiento y Sistema Penitenciario. En Sanmartín (coord.), El laberinto de la violencia: causas, tipos y efectos, (pp.331-341). Barcelona: Ariel.

Redondo, S.; Pozuelo, F. y Ruíz, A. (2007). El tratamiento en las prisiones: Investigación internacional y su situación en España. En A. I. Cerezo y E. García (coords.) La prisión en España: una perspectiva criminológica,(pp. 175-212). Granada: Comares.

Reglamento Penitenciario aprobado por RD 190/1996 de 9 de febrero.

Ruiz Pérez, J. I. (2006). Clima emocional y sobreocupación en prisión: una evaluación mediante informantes clave. Suma Psicológica, 13 (2), 159-172.

Ruiz Pérez, J.I: (2009). La experiencia del encarcelamiento: una perspectiva psicosocial. En J. I. Ruiz Pérez, L. Rodríguez, (dirs.) y E. Meluk, (coord.), Estado del arte en psicología: aportes desde la psicología jurídica y clínica al contexto penitenciario (Documento de Trabajo). Recuperado de http://extension.upbbga.edu.co/inpec2009/Estudiosprimeraparte/ areasdisciplinares/ psicologia.pdf. 
Topa, G. y Morales, J. (2005). Determinantes específicos de la satisfacción laboral, el Burnout y sus consecuencias para la salud: un estudio exploratorio con funcionarios de prisiones. International Journal of Psychology and Psychological Therapy, 5 (1), 73-83.

Trillo, M. (2008). Los efectos de la privación de la libertad en la subjetividad (y algunos modos de resistencia). Clepios: revista de residentes de salud mental, XIII (3), 100-103.

Valderrama, P. (2012). La micropolítica de la función reeducadora en prisión. Revista de Educación, 360, 69-90. Recuperado de http://www.mecd.gob.es/revista-de-educacion/numeros-revista-educacion/ultimo-numero/re360/re360_05.html

Viegas, F. (2009).La reja en la cabeza. Etnografía, representaciones, experiencias y mediaciones en torno al impacto de la cárcel en personas con libertad ambulatoria. Revista Question, 1, 23. Recuperado de http://perio.unlp.edu.ar/ ojs/index.php/question/ article/view/837/738

\section{Notas}

${ }^{1}$ Este trabajo se inserta en el marco del Plan Nacional de Investigación, el Proyecto l+D+l denominado 'Mujeres reclusas drogodependientes y su reinserción social. Estudio socioeducativo y propuestas de acción' [EDU2009-13408] financiado por el Ministerio de Economía y Competitividad (MICCIN) del gobierno español. Asimismo, cabe agradecer el apoyo y participación de la Secretaría General de Instituciones Penitenciarias, la Consejería de Justicia de la Generalidad de Cataluña, a cada uno de los Centros de cumplimiento de pena estudiados y, de todas las mujeres reclusas.

2 Agradecimiento especial a Araceli Fernández Iglesias por su activa participación en el equipo de trabajo.

3 Régimen ordinario es el aplicado a penados de segundo grado, a los penados sin clasificar y detenidos y presos (Reglamento Penitenciario, 1996).

4 Un centro abierto alberga a penados clasificados en tercer grado (Reglamento Penitenciario, 1996)

5 Ítems50, 51.3, 51.4, 51.5, 51.7, 51.8, 51.10, 55.1.a y 55.1.b y 55 del cuestionario a mujeres reclusas. Ítems 166, 167,186 y 188 de la entrevista a mujeres reclusas. Ítems 29, 30 y 31 del cuestionario a profesionales.

\section{Dirección de los autores}

Ma del Mar García-Vita. Universidad de Granada. Facultad de Ciencias de la Educación. Campus de Cartuja, 1807ı, Granada (Spain)

Miguel Melendro Estefanía. Universidad Nacional de Educación a Distancia. Facultad de Ciencias de la Educación. Departamento de Teoría de la Educación y Pedagogía Social. c/ Juan del Rosal, 14. 28030. Madrid (Spain)

Correo electrónico: marvita19@hotmail.com,mmelendro@edu.uned.es

Fecha de recepción del artículo: 7.2.2013

Fecha de revisión del artículo: 6.3.2013

Fecha de aceptación final: 22.4.2013

\section{Cómo citar este artículo}

García-Vita, M. M., Melendro Estefanía, M. (2013). El ambiente en prisión: La atención recibida por las reclusas y las relaciones intramuros. Pedagogía Social. Revista Interuniversitaria, 22, pp-pp. 43-56

[ 56 ] Ma DEL MAR GARCÍA-VITA, MIGUEL MELENDRO ESTEFANÍA

SIPS - PEDAGOGIA SOCIAL. REVISTA INTERUNIVERSITARIA [1139-1723 (2013) 22, 43-56] TERCERA ÉPOCA 